\title{
Fixed-time synchronization of coupled memristive neural networks with multi-links and application in secure communication
}

\author{
Hui Zhao ${ }^{1}$, Aidi Liu ${ }^{1}$, Qingjie Wang ${ }^{1}$, Mingwen Zheng ${ }^{2}$, Chuan $\mathrm{Chen}^{3}$, Baozhu $\mathrm{Li}^{1}$, and \\ Sijie Niu ${ }^{1}$ \\ ${ }^{1}$ University of Jinan \\ ${ }^{2}$ Shandong University of Technology \\ ${ }^{3}$ Qilu University of Technology (Shandong Academy of Sciences)
}

March 13, 2021

\begin{abstract}
This paper is devoted to investigating the issues of fixed-time synchronization of coupled memristive neural networks with multilinks (MCMNN). Based on the fixed-time stability criterion and the upper bound estimate formula for the settling time, we propose a secure communication scheme. The network with multi-links performance and coupled form increase the complexity of network topology and the unstable of systems, which improve security of communication in the aspect of encrypt the plaintext signal. We design a proper controller and build the Lyapunov function, several effective conditions are obtained to achieve the fixed-time synchronization of MCMNN. Moreover, the settling times can be estimated for fixed-time synchronization without depending on any initial values. Meanwhile, the plaintext signals can be recovered according to the fixed-time stability theorem. Finally, numerical simulations are given to verify the effectiveness of the theoretical results in fixed-time synchronization of MCMNN, and an example of a secure communication scheme is given to show the usability and superiority based on fixed-time stability theorem.
\end{abstract}

\section{Hosted file}

manuscript.pdf available at https://authorea.com/users/401389/articles/513484-fixed-timesynchronization-of-coupled-memristive-neural-networks-with-multi-links-and-applicationin-secure-communication 